\title{
Reduced Blood-brain Barrier Penetration of Sarecycline Relative to Minocycline in Rats Corresponds with Lipophilicity and Low Vestibular Side Effects
}

Linda Stein-Gold ${ }^{1}$, Angela Moore ${ }^{2,3}$, S. Ken Tanaka ${ }^{4}$, Jodi L. Johnson ${ }^{5}$, Ayman Grada ${ }^{6}$

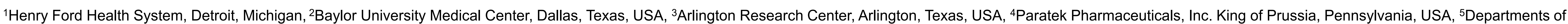
Dermatology and Pathology, Feinberg School of Medicine, Northwestern University, USA, ${ }^{6}$ R\&D and Medical Affairs, Almirall (US), Exton, Pennsylvania, USA Email: Grada@bu.edu

\section{Introduction}

Sarecycline is an FDA-approved narrow-spectrum tetracycline-class oral antibiotic specifically designed for the treatment of moderate-to-severe acne vulgaris.

Doxycycline and minocycline have historically been reported with side effects of dizziness, vertigo, or tinnitus.

Pooled data from 2 Phase III randomized controlled trials $(n=2002)$ and a 40-week open-label extension study $(n=483)$ for sarecycline reported low rates of vestibular events (dizziness $(\leq 0.5 \%)$, vertigo $(0 \%)$, and tinnitus $(0 \%)$ ).

We sought to investigate penetration of the blood-brain barrier of sarecycline relative We sought to investigate penetration of the blood-brain barrier of sarecycline relative
to minocycline in a rat model and the relative lipophilicity of sarecycline compared to to minocycline in a rat model
minocycline and doxycycline.

\section{Methods}

Table 1. Blood-brain barrier penetration: Rats (pre-cannulated, jugular vein) were dosed with IV sarecycline or minocycline at a total dose of $1.0 \mathrm{mg} / \mathrm{kg}$. Rats were fasted overnight (about 16 hours) prior to dosing and access to food was restored 2 hours after dosing. Animals were euthanized via $\mathrm{CO}_{2}$ and whole blood (via heart puncture) and brain were collected from 2 rats at each of the following time points: 1,3 and $6 \mathrm{hr}$ post dosing.

Table 2. Lipophilicity: The octanol/water distribution coefficients $(\log D)$ of sarecycline, minocycline, and doxycycline were measured using the shake flask method at $\mathrm{pH} 5.5$ and 7.4 at $25^{\circ} \mathrm{C}$.
Results - Table 1. Unlike minocycline, sarecycline was not detectable in the brain in rats

\begin{tabular}{|c|c|c|c|c|}
\hline Time & Mcn-pl & Scn-pl & Mcn-br & Scn-br \\
(hours) & $\mu \mathrm{g} / \mathbf{m L}$ & $\mu \mathrm{g} / \mathbf{m L}$ & $\mu \mathrm{g} / \mathbf{g}$ & $\mu \mathrm{g} / \mathbf{g}$ \\
\hline 1 & 0.333 & 0.460 & 0.074 & $\mathrm{BLQ}$ \\
\hline 3 & 0.174 & 0.217 & 0.139 & $\mathrm{BLQ}$ \\
\hline 6 & 0.077 & 0.049 & 0.068 & $\mathrm{BLQ}$ \\
\hline
\end{tabular}

$\mathrm{PI}=$ plasma, $\mathrm{Br}=$ brain, $\mathrm{Mcn}=$ minocycline, $\mathrm{Scn}=$ sarecycline Limit of quantitation (LOQ) (plasma) $=0.025 \mathrm{\mu g} / \mathrm{mL}$, LOQ (brain) $=0.05$ $\mu \mathrm{g} / \mathrm{g} ; \mathrm{BLQ}-$ Below the limit of quantitation

Results - Table 2. Sarecycline has slightly lower lipophilicity than minocycline and doxycycline

\begin{tabular}{l|l|l} 
Compound & pH 5.5 & pH 7.4
\end{tabular}

\begin{tabular}{|l|l|l}
\hline Sarecycline $\mathrm{HCl}$ & $-0.16 \pm 0.01$ & $-0.26 \pm 0.01$ \\
\hline
\end{tabular}

\begin{tabular}{|l|l|l|}
\hline Doxycycline $\mathrm{HCl}$ & $-0.00 \pm 0.02$ & $-0.18 \pm 0.03$ \\
\hline
\end{tabular}

Minocycline $\mathrm{HCl}$

$0.09 \pm 0.02$

$0.12 \pm 0.02$

Octanol/water distribution coefficients of sarecycline $\mathrm{HCl}$, minocycline $\mathrm{HCl}$, and doxycycline $\mathrm{HCl}$ at $25^{\circ} \mathrm{C}$. The numbers after \pm represent

standard deviations obtained from triplicate samples.
Discussion - Table 3. Vestibular adverse events were low in Phase 3 efficacy and safety studies for sarecycline

\begin{tabular}{l|l|l}
\hline Vestibular effects & Sarecycline $(n=994)$ & Placebo $(n=996$
\end{tabular}

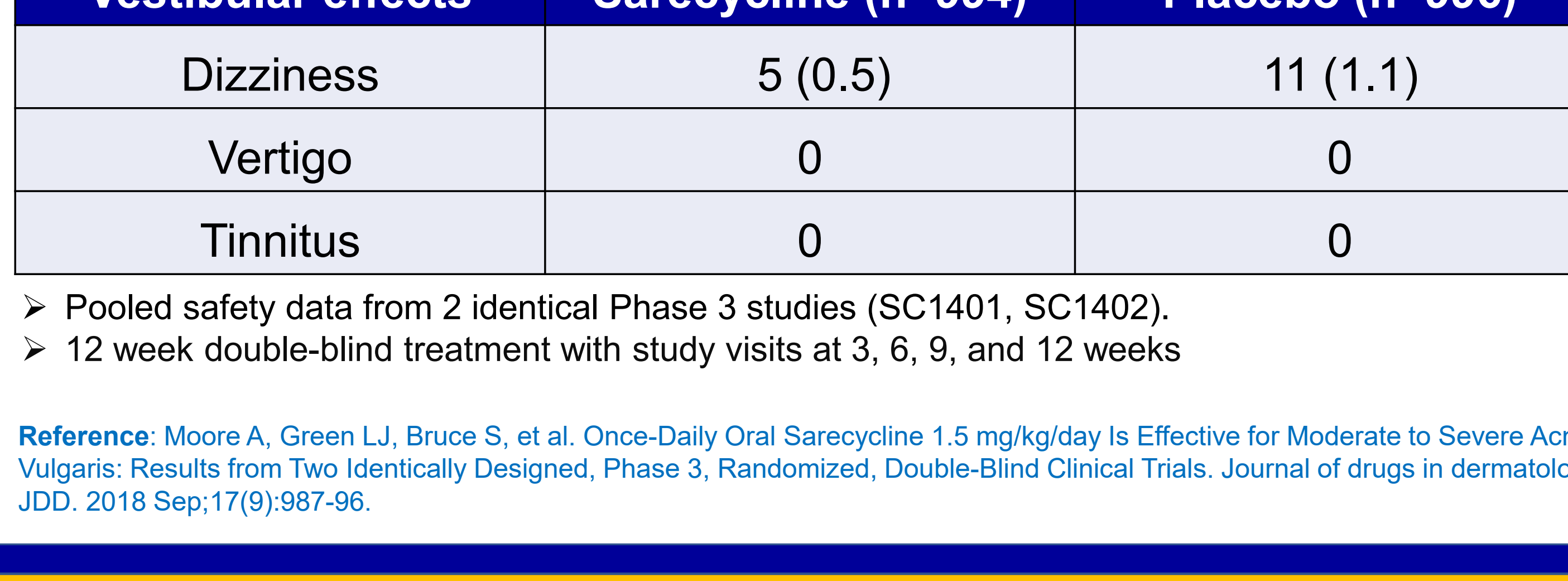

Discussion - Table 4. Vestibular adverse events were low in an openlabel long-term safety study for sarecycline

\begin{tabular}{|c|c|c|c|}
\hline $\begin{array}{c}\text { Vestibular } \\
\text { effects }\end{array}$ & $\begin{array}{c}\text { Placebol } \\
\text { Sarecycline } \\
(\mathbf{n = 2 3 6 )}\end{array}$ & $\begin{array}{c}\text { Sarecyclinel } \\
\text { Sarecycline } \\
(\mathbf{n = 2 4 7 )}\end{array}$ & Total (n=483) \\
\hline Dizziness & $1(0.4)$ & $1(0.4)$ & $2(0.4)$ \\
\hline Vertigo & 0 & 0 & 0 \\
\hline Tinnitus & 0 & 0 & 0 \\
\hline
\end{tabular}

$>$ Patients from previous 12 week Phase 3 studies received once daily sarecycline for up to 40

weeks.

Reference: Pariser DM, Green LJ, Lain EL, et al. Safety and Tolerability of Sarecycline for the Treatment of Acne Vulgaris: Results
from a Phase III, Multicenter, Open-Label Study and a Phase I Phototoxicicity Study. Journal of Clinical and Aesthetic Dermatology.
2019:12(11) E53-E62

\section{Conclusions}

Sarecycline's inability to cross the blood-brain barrier compared to minocycline corresponds with sarecycline's lower lipophilicity and may explain the low rate of vestibular adverse events observed in sarecycline's clinical trials. 